\title{
Stability of the interaction between two sand dunes in an idealized laboratory experiment
}

\author{
Karol A. Bacik, ${ }^{1, *}$ Colm-cille P. Caulfield, ${ }^{2,1}$ and Nathalie M. Vriend ${ }^{2,1,3}$ \\ ${ }^{1}$ Department of Applied Mathematics \& Theoretical Physics, \\ University of Cambridge, Centre for Mathematical Sciences, \\ Wilberforce Road, Cambridge CB3 OWA, United Kingdom \\ ${ }^{2}$ BP Institute, University of Cambridge, Madingley Rise, \\ Madingley Road, Cambridge CB3 OEZ, United Kingdom \\ ${ }^{3}$ Department of Earth Sciences, University of Cambridge, \\ Downing Street, Cambridge CB2 3EQ, United Kingdom
}

(Dated: August 25, 2021)

\begin{abstract}
Sand dunes, which arise spontaneously due to the dynamical interplay between a sedimentary interface and a fluid flow, are one of the most famous examples of emergence in a geological system. The large scale organisation of a dune field is believed to be controlled by pairwise (either remote or direct) dune-dune interactions. Recent studies have shown that remote long-range feedback is closely related to the turbulent wake structure forming downstream of a dune. Here we study the stability of an idealised two-dune system arising as a consequence of such remote, wake-induced interactions. The system is realised in a subaqueous quasi-2D laboratory experiment and the results are compared with a qualitative dynamical systems model. Despite its simplicity, the system exhibits rich dynamical behaviour. In particular we show that, depending on the parameter regime, the dune-dune feedback can either stabilise or destabilise the symmetric dune configuration, and we demonstrate the existence of an asymmetric attracting state coupling dunes of different sizes.
\end{abstract}

Sand dunes often form vast collectives known as dune fields [1-3], which continually evolve due to erosion and deposition induced by the flow of the overlaying fluid [48]. The migration of terrestrial dunes is related to desertification and poses a threat to human-made infrastructure, both in deserts and under water $[5,9,10]$. By studying extraterrestrial dunes we can also learn about the weather conditions on other planets $[11,12]$. Dune field modelling, therefore, is a pressing need, but it is also challenging as full resolution of all the relevant fluid dynamics is practically impossible with the current computational resources. Consequently, numerical studies typically rely on empirical parametrisations. The most radical reduced models abstract a sparse dune field as a population of discrete agents interacting with their surroundings. Previous models of this kind focused on collisional interactions leading to billiard-like dynamics [1, 3, 1320]. However, recent experimental and observational studies revealed that dunes can also interact over sizable distances without necessarily directly colliding [2125]. Therefore, while the agent-based models still remain attractive, their parametrisation stands in need for a revision.

In this letter, we study the stability of an experimentally realised quasi-2D two-dune system [26-29], which can be regarded as a paradigm of remote dune-dune interactions. In our experiments we use a narrow annular flume (see Fig. 1) which gives the unique opportunity to impose periodic boundary conditions in a laboratory experiment [24] (for technical details see Supplementary Material, including Refs. [30, 31]). The closest

\footnotetext{
*kab81@cam.ac.uk
}

natural analogue of our experimental dunes are spanwiseinvariant transverse dunes, but they can also be interpreted as streamwise transects of more complex bedforms [32]. Quasi-2D bedforms have received significant attention in the theoretical literature, which allows us to place our experimental results in the context of wellestablished theories [32, 33].

For quasi-2D dunes confined in a channel of width $W$, the changes in sediment topography $h(x, t)$ can be related to the sand flux $q(x, t)\left[M T^{-1}\right]$ through the Exner equation $[4,33]$

$$
\frac{\partial h}{\partial t}+\frac{1}{\rho} \frac{\partial q}{\partial x}=0,
$$

where

$$
\rho=\rho_{p} \eta W
$$

is the effective density of the quasi-2D bedform, $\rho_{p}$ is the actual sediment density, and $\eta$ is the bed packing fraction. As a corollary, a steady-shape migrating dune moves at rate

$$
c=\frac{q^{c}-q^{i}}{\rho h^{c}},
$$

where $q^{i}$ is the upstream sediment in-flux, $q^{c}$ is the sediment flux at dune's crest, and $h^{c}$ is the crest height [4, 32,33 . If the dune's cross-section remains self-similar in shape, we can also assume

$$
h^{c}=\sqrt{\frac{k m}{\rho}},
$$

where $m$ is the mass of the dune and $k$ is a proportionality constant. 

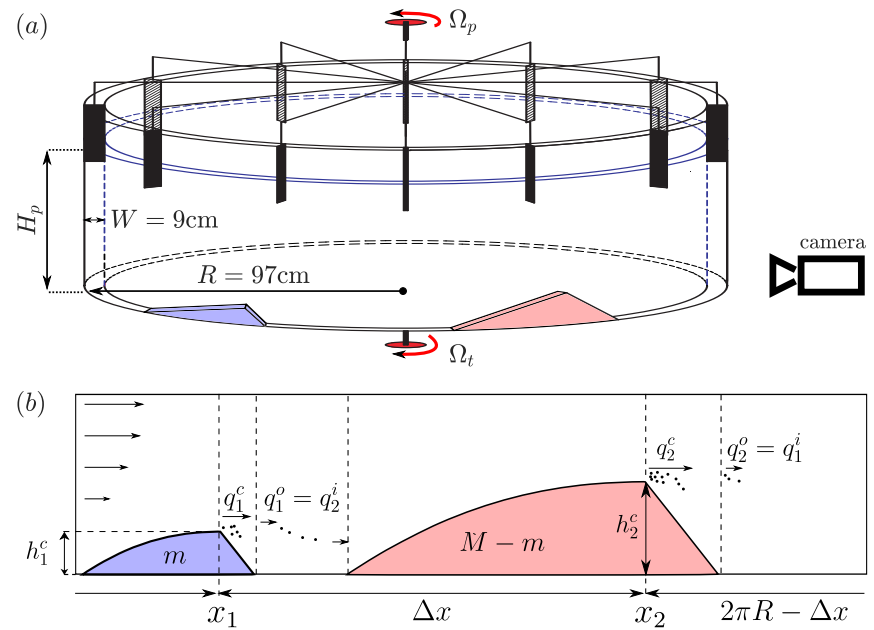

Figure 1. (a) Experimental set-up. Annular channel of outer radius $R$ and a working section of width $W$, filled with water up to $H_{w}=45 \mathrm{~cm}$. The dominant flow, induced by paddle motion, is from the blue (upstream) dune to the red (downstream) dune. Paddle tip position $H_{p}$, as well as the table rotation rate $\Omega_{t}$ and the paddle rotation rate $\Omega_{p}$ vary between experiments (cf. Table I). (b) Quasi-2D twodune problem with dunes of masses $m_{1}=m, m_{2}=M-m$ and crests of height $h_{1,2}^{c}$, located at $x_{1,2}$. The evolution of the system can be related to respective (sediment) crest fluxes $q_{1,2}^{c}$, in-fluxes $q_{1,2}^{i}$, and out-fluxes $q_{1,2}^{o}$.

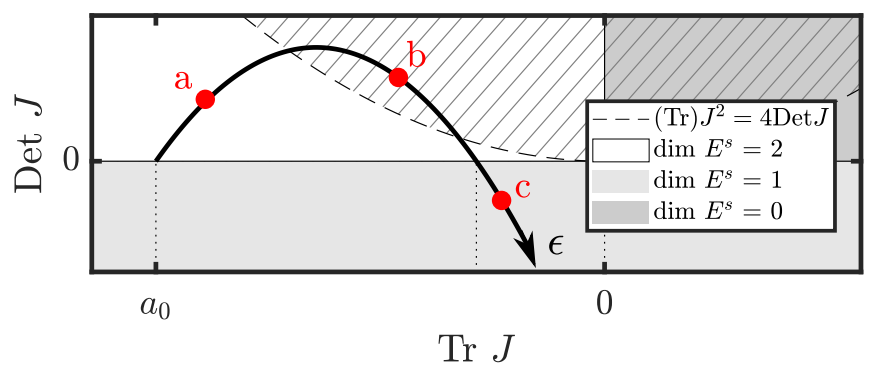

Figure 2. Trace-determinant plane. Shadings represent the dimension of the stable manifold $E_{s}$ and the hashed region corresponds to oscillatory solutions. All else being fixed, as the sand-trapping efficiency $\epsilon$ increases we trace a parabola. Hence, one possible bifurcation scenario, depicted by the solid black line, is stable node (a) $\rightarrow$ stable focus (b) $\rightarrow$ stable node $\rightarrow$ saddle (c).

We shall now specialise to the problem of a two-dune system in a periodic domain. In a reduced model, this system is described by four variables $\left(x_{1}, x_{2}, m_{1}, m_{2}\right)$ [34], but we can reduce its dimensionality even further. Firstly, due to rotational symmetry of the system, we will only focus on the relative dune separation

$$
\Delta x=x_{2}-x_{1} .
$$

Secondly, we will assume that the inter-dune particle transit times are instantaneous, so the respective in- and out-fluxes are equal and

$$
m_{2}=M-m_{1},
$$

where $M$ is the total mass of sediment in the flume. Thus, the state of our system is fully described by separation $\Delta x$ and the mass $m=m_{1}$ coupled by the following equations:

$$
\begin{gathered}
\frac{d(\Delta x)}{d t}=\frac{q_{2}^{c}-q_{1}^{o}}{\sqrt{k \rho(M-m)}}-\frac{q_{1}^{c}-q_{2}^{o}}{\sqrt{k \rho m}}, \\
\frac{d m}{d t}=q_{2}^{o}-q_{1}^{o} .
\end{gathered}
$$

In order to parameterise the remote dune-dune interactions, we will assume that the respective fluxes are modulated by the upstream conditions, i.e.

$$
\begin{aligned}
q_{1}^{c} & =q_{0}\left(1+f^{c}(2 \pi R-\Delta x,(M-m) / \rho)\right) \\
q_{1}^{o} & =\epsilon q_{0}\left(1+f^{o}(2 \pi R-\Delta x,(M-m) / \rho)\right) \\
q_{2}^{c} & =q_{0}\left(1+f^{c}(\Delta x, m / \rho)\right) \\
q_{2}^{o} & =\epsilon q_{0}\left(1+f^{o}(\Delta x, m / \rho)\right)
\end{aligned}
$$

Parameter $\epsilon$ is the 'sand trapping efficiency' [34, 35], quantifying how much of the sand swept away from one dune's crest is trapped by the flow recirculation in the lee [36-38]. Lacking exact experimental measurements, we shall refrain from suggesting any particular form for the interaction functions $f^{c}$ and $f^{o}$. Nevertheless, based on previous experiments [24] we can postulate three properties:

1. $f^{c}, f^{o} \geq 0$, as the turbulent fluctuations generically enhance sediment flux;

2. $\frac{\partial f^{c}}{\partial y_{1}}\left(y_{1}, y_{2}\right), \frac{\partial f^{o}}{\partial y_{1}}\left(y_{1}, y_{2}\right) \leq 0$, as feedback is expected to decay away from the upstream dune;

3. $\frac{\partial f^{c}}{\partial y_{2}}\left(y_{1}, y_{2}\right), \frac{\partial f^{o}}{\partial y_{2}}\left(y_{1}, y_{2}\right) \geq 0$, as feedback is expected to be stronger for larger obstacles.

Expressions (9) provide a closure for the system (7)(8). For mathematical convenience, we shall now replace its dimensional variables with non-dimensional

$$
\Delta \theta=\Delta x / R, \quad \mu=m / M, \quad \tau=t \frac{\Omega_{t o t} R \rho d}{M},
$$

where $d$ is the mean particle diameter and $\Omega_{t o t} R=\mid \Omega_{p}-$ $\Omega_{t} \mid R$ provides the velocity scale (c.f. Fig. 1(a)).

Note that $(\Delta \theta, \mu) \in[0,2 \pi) \times[0,1]$ and, due to periodic boundary conditions, the system possesses a symmetric fixed point $S=(\Delta \theta=\pi, \mu=1 / 2)$. Its linear stability properties are (naturally) controlled by the eigenvalues of the Jacobian matrix $J$, but in 2D they can be inferred from the values of the trace and determinant of $J$, without detailed calculation of the eigenvalues themselves. In our system,

$$
\operatorname{Tr} J=a_{0}+a_{1} \epsilon,
$$

and

$$
\operatorname{Det} J=b_{1} \epsilon+b_{2} \epsilon^{2},
$$



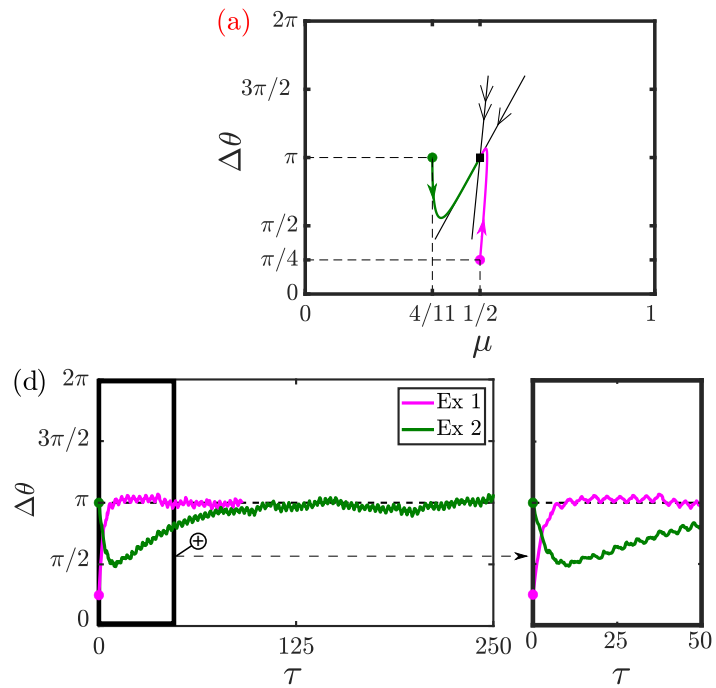
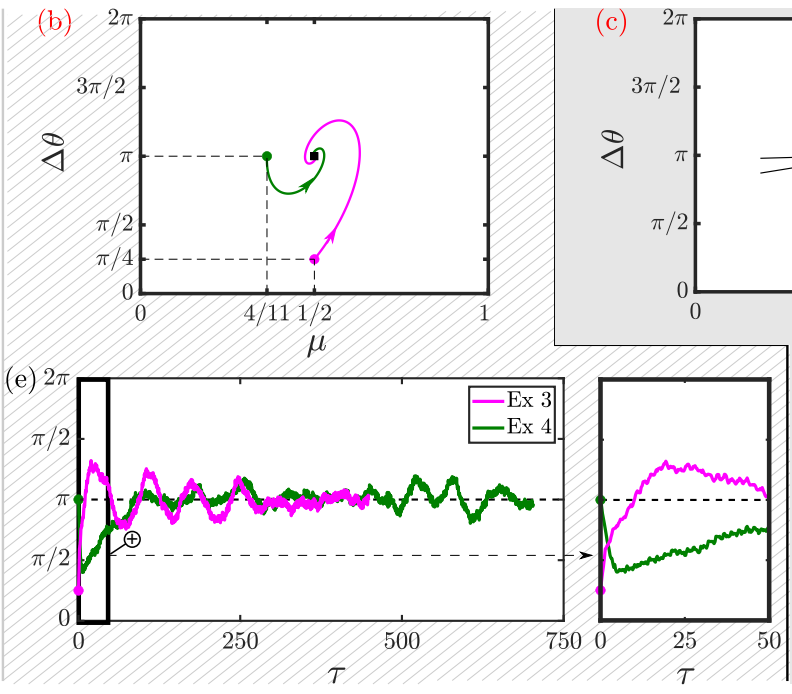
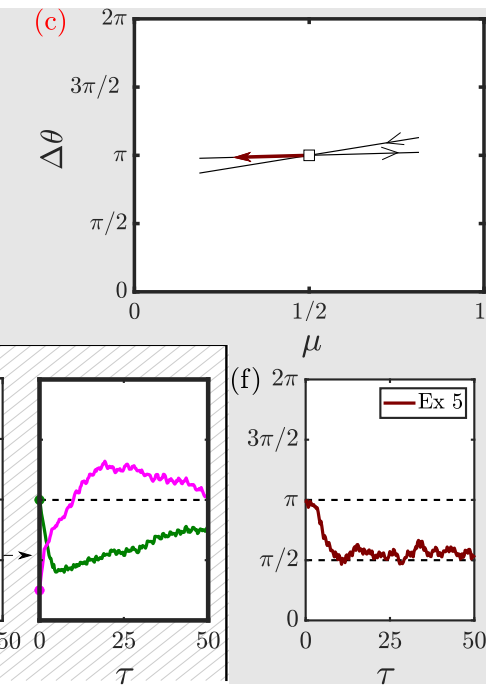

Figure 3. Linear stability of the symmetric fixed point $S$. The top panels shows illustrative theoretically predicted phase space diagrams for (a) a stable node, (b) a stable focus, (b) a saddle. The bottom panels show corresponding experimentally measured $\Delta \theta(\tau)$. (d) Under quiescent flow conditions $S$ behaves as a stable node. The timecourse in magenta corresponds to a pure $\Delta \theta$-perturbation and the timecourse in green was initialised with a pure $\mu$-perturbation. (e) Under intermediately turbulent conditions the dynamics exhibits clear oscillatory features. (f) When the flow is sufficiently turbulent, the system diverges from the symmetric fixed point $S$ and moves towards asymmetric attractor $A$.

where $a_{0}, b_{2}<0$ and $b_{1}>0$ (see Supplementary Material). For fixed $a_{i}$ and $b_{i}$, equations (11)-(12) parametrically define a parabola in the trace-determinant plane. Thus, as $\epsilon$ increases, we expect the fixed point to change its linear character. In particular, for $\epsilon \ll 1, S$ is a stable node and in the limit $\epsilon \rightarrow \infty$, it becomes a saddle. In the intermediate regime, depending on the exact values of $a_{i}$ and $b_{i}$, there is also a possibility of both damped and amplified oscillatory solutions (Fig. 2).

This simple analysis can be used to explain our experimental results, which show that the stability properties of $S$ change as we vary the flow parameters. The relationship between the flow conditions and $\epsilon$ can be understood in terms of the turbulence intensity $\mathcal{I}$. We should emphasise however, that due to experimental limitations we cannot measure either $\mathcal{I}$ or $\epsilon$, so our arguments remain purely qualitative. To begin with, recall that the sand trapping efficiency $\epsilon$ can be interpreted as a ratio of fluxes $\epsilon \sim q^{o} / q^{c}$. As previously noted, $q^{o}$ can be increased by the turbulent gusts, which allow single sand particles to bypass the recirculation region [24, 39-41]. Therefore, it is plausible that $q^{\circ}$, and therefore $\epsilon$ increases as $\mathcal{I}$ increases. Indeed, even though the turbulent fluctuations can increase $q^{c}$ as well, $q^{c}$ does not critically rely on these fluctuations in the way the remote transport to the other dune (and hence $q^{\circ}$ ) does. As far as the experiment is concerned, $\mathcal{I}$ can be increased in at least two ways. Firstly, by increasing the net driving rotation rate $\Omega_{t o t}=\left|\Omega_{p}-\Omega_{t}\right|$ (i.e. the differential angular speed between the paddle and the turntable, see Fig. 1(a)), which impacts the channel-based Reynolds number. Secondly, by reducing $H_{p}$, which is the the distance between the channel's floor and lower tip of the paddle which sheds

\begin{tabular}{c|c|c|c|c|c|c} 
& $H_{p}(\mathrm{~cm})$ & $\Omega_{\text {tot }}(\mathrm{rpm})$ & $\Omega_{t}(\mathrm{rpm})$ & $\Omega_{p}(\mathrm{rpm})$ & $\Delta \theta(0)$ & $\mu(0)$ \\
\hline \hline Ex1 & 38.5 & 13.5 & -5.10 & 8.40 & $\pi / 4$ & $1 / 2$ \\
\hline Ex2 & 38.5 & 13.5 & -5.10 & 8.40 & $\pi$ & $4 / 11$ \\
\hline Ex3 & 34 & 13.5 & -5.40 & 8.10 & $\pi / 4$ & $1 / 2$ \\
\hline Ex4 & 34 & 13.5 & -5.40 & 8.10 & $\pi$ & $4 / 11$ \\
\hline Ex5 & 24 & 15.5 & -6.30 & 9.20 & $\pi$ & $1 / 2$ \\
\hline Ex6 & 24 & 13.5 & -5.85 & 7.65 & $41 \pi / 45$ & $10.5 / 22$ \\
\hline \hline
\end{tabular}

Table I. Experiments. Flow boundary conditions as well as the initial condition for the six experiments presented in this letter. The counter-rotation ratio $\left|\Omega_{p} / \Omega_{t}\right|$ is tuned empirically to ensure a quasi-2D profile of the sedimentary bed [42].

intense turbulent structures. If $H_{p}$ is large, most of the eddies dissipate before they reach the floor, but if $H_{p}$ is small, they impinge on the surface of a dune with more intensity. These two numbers $\left(\Omega_{t o t}\right.$ and $\left.H_{p}\right)$ are the only flow parameters which vary across the six experiments presented in this letter and which are summarised in Table I. The experiments could last up to 80 hours, so for technical reasons they had to be paused several times. However, through independent validation experiments, we are confident that stopping and restarting an experiment has no significant dynamical effect after a spin-up time of the order of sixty seconds.

Experiments 1 and 2 (Fig. 3(d)) were conducted under the relatively quiescent, but still definitely turbulent $\left(R e_{\text {dune }} \sim 10^{4}\right)$, flow conditions, with $H_{p}=38.5 \mathrm{~cm}$, so that the blade protrudes above the free surface. Experiment 1 corresponds to a pure $\Delta \theta$-perturbation and Experiment 2 started from a $\mu$-perturbation away from the 


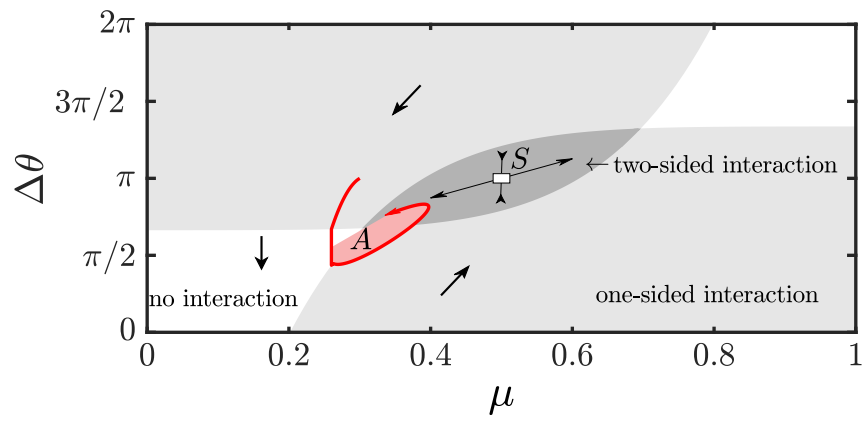

Figure 4. Asymmetric attractor. Schematic of the phase space explaining the physical nature of the asymmetric attractor $A$ indicating distinct regions of one-sided, two-sided or no interaction. The red line corresponds to a thought experiment trajectory, which can be used to infer the existence of an attracting forward-invariant region.

symmetric fixed point $S$. In both cases, the system eventually converges to $S$. Indeed, even though we cannot measure dune mass at all times without disrupting the experiment, it has been verified that the masses of the two dunes are virtually equal at the end of the experiment. Based on the character of the $\Delta \theta$ time evolution, we conclude that under these flow conditions $S$ behaves as a stable node, corresponding to the dynamical regime $\epsilon \ll 1$. The time-scale separation $\left(\frac{d \mu}{d \tau} / \frac{d(\Delta \theta)}{d \tau}=O(\epsilon)\right)$ is further corroborated by the slow-fast characteristics apparent in Experiment 2. Here we observe quick convergence towards the slow manifold (with $\frac{d(\Delta \theta)}{d \tau} \approx 0$ ) and a slow mass rearrangement.

As demonstrated in Figure 3(e), the dynamics is qualitatively different when the paddle is lowered down further. This time, we observe manifest oscillations around $S$. Thus, at a linear level $S$ acts as a focus (most likely stable), which would be consistent with intermediate values of $\epsilon$. Nevertheless, at the nonlinear level, the system exhibits very interesting excitable features. Indeed, Experiment 4 demonstrates that the oscillations can be also spontaneously induced by the intrinsic system noise originating from turbulence and flume imperfections.

Finally, under the most turbulent conditions (Fig. 3(f)), $S$ becomes unstable. Here, the experiment was initiated exactly at $S$, but the system quickly moved away from the symmetric configuration and converged to an asymmetric attractor $A \approx(\pi / 2,4 / 11)$. The instability of $S$ agrees with our linear stability analysis in the regime $\epsilon=O(1)$. Nevertheless, this calculation clearly cannot explain the existence of the asymmetric attractor $A$.

The nature of the asymmetric attractor $A$ can be understood through consideration of the schematic shown in Fig. 4. So far, our analysis has been based upon the implicit assumption that the domain length is small enough, so that the dune-dune feedback is bidirectional. This assumption is manifestly true for the symmetric fixed point $S$, but it may not be true for all the configurations. In
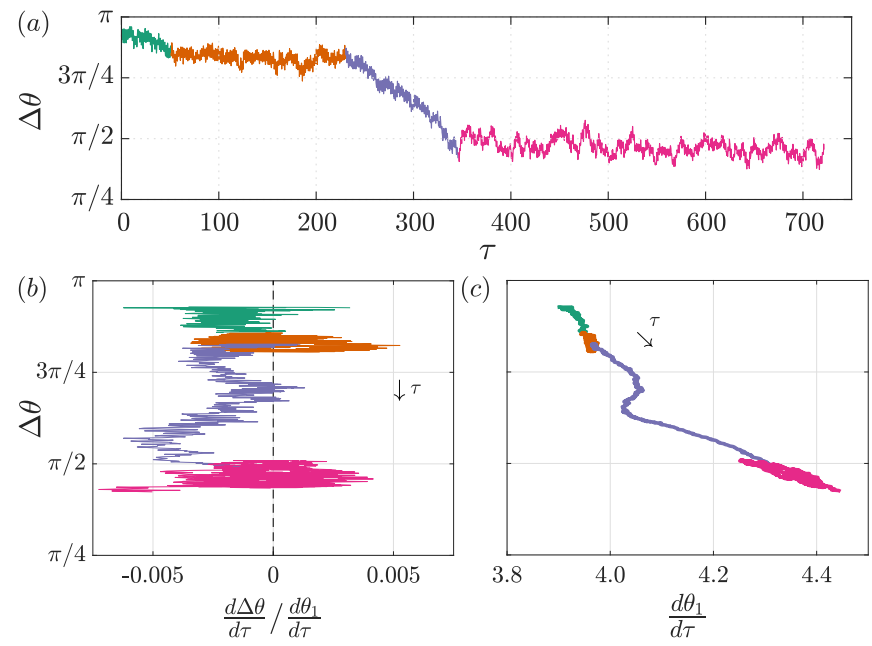

Figure 5. Transition. (a) Timecourse $\Delta \theta(\tau)$ of Experiment 6 starting from a small finite-size perturbation of $S$, which is non-linearly unstable. (b) Differential speed of individual dunes for the same experiment. (c) Migration speed of the leading dune (c.f. Fig. 1(b)). Different colours denote different time periods.

particular, for sufficiently small $\mu$, there may be a range of dune separations $\Delta \theta$ where the dunes are effectively decoupled (shown in white in Fig. 4). If that is the case, $\frac{d \mu}{d \tau}=0$ and the migration rate depends only on the dune size, so that $\frac{d(\Delta \theta)}{d \tau}<0$ for $\mu<\frac{1}{2}$ and $\frac{d(\Delta \theta)}{d \tau}>0$ for $\mu>\frac{1}{2}$. On the other hand, in the double limit $\Delta \theta \rightarrow 0, \mu \rightarrow 1$ (or symmetrically $\Delta \theta \rightarrow 2 \pi, \mu \rightarrow 0$ ), which corresponds to a small upstream dune 'chasing' a big downstream dune, we expect a unidirectional feedback (shaded light grey in Fig. 4). If the small dune imposes sufficiently strong repulsion to prevent a collision, over a long time scale, the bigger dune is drained of mass, so the system is attracted towards the two-sided interaction region (shaded dark grey in Fig. 4). Thus, by considering a bounding trajectory starting in one of the one-sided interaction regions (red in Fig. 4), we conclude that the instability of $S$ implies the existence of a forward-invariant region $A$, which by the Poincaré-Bendixson theorem contains a simple attractor [43]. Nevertheless, unless the details of the slow manifold are resolved, it is not guaranteed that $A$ possesses a linearly stable fixed point. Indeed, we observe the dynamics around $A$ is rather noisy (Fig. 3(f) and Fig. 5(a)). These large amplitude fluctuations may be ascribed to the fact that the small dune is more sensitive to mass perturbations, but they may also hint about the possible lack of a single attracting fixed point.

Figure 5 shows an experiment near the bifurcation point where $S$ is marginally stable. It is important to appreciate that, due to slow transients, it is difficult to determine if $S$ is indeed linearly stable at this particular point, but it is clear that it certainly is unstable to finitesize (yet still relatively small) perturbations. The experiment starts with a small perturbation, approximately in 
the direction of the slow manifold, and it first moves toward a metastable intermediate state $I$ with $\Delta \theta \approx 0.8 \pi$. It is plausibly related to some relatively short-lived (in parameter space) fixed point which is born when $S$ looses stability. Nevertheless, the system eventually drifts away from $I$ and converges to the asymmetric state $A$, where it remains for the last 350 non-dimensional time units, i.e. about $40 \mathrm{~h}$, of the experiment. Figure 5(b) shows the difference in the migration rate between the two dunes throughout the experiment. It is important to realise that the differential velocity is in fact rather small, in that the migration rates of the two dunes agree to at least two significant figures. Nevertheless, the small excess of speed of the upstream dune over time accumulates to a significant change in $\Delta \theta$. Figure 5 (c) shows also that the equilibrium migration rate is faster in $A$ than in $S$, which agrees with the expectation that the migration rate should be dictated by the smallest dune in the system.

Our experimental set-up clearly lacks the complexity of any natural dune field, but the agent-based framework is sufficiently general to draw a robust analogy between our experiments and natural dunes. Thus, we would argue that these results form an essential first step towards understanding the role of remote dune-dune interactions by demonstrating how such interactions can qualitatively change the long-time coupled dynamics through a remote sand transport feedback. As we have seen, this feedback can have both stabilising and destabilising effects. Of particular interest is also the evidence of the existence of an asymmetric attracting state, where dunes of different sizes (and different downstream separations) couple in a balanced fashion over very long times. The next step is to consider the implications of this feedback for a longer train of dunes and the 3D phenomena, such as fragmentation [21, 25, 44, 45] or calving [18].

\section{ACKNOWLEDGMENTS}

$\mathrm{KAB}$ acknowledges the support of a sponsored $\mathrm{PhD}$ studentship from Schlumberger Cambridge Research. NMV is supported by a Royal Society University Research Fellowship URF/R1/191332. The authors thank Professor Stuart Dalziel and the technical team of the GK Batchelor laboratory as well as Dr Paul Jarvis.
[1] P. Hersen, K. H. Andersen, H. Elbelrhiti, B. Andreotti, P. Claudin, and S. Douady, Phys. Rev. E 69, 011304 (2004).

[2] G. Kocurek and R. C. Ewing, Geomorphology 72, 94 (2005).

[3] M. Génois, S. C. du Pont, P. Hersen, and G. Grégoire, Geophys. Res. Lett. 40, 39093914 (2013).

[4] F. Charru, B. Andreotti, and P. Claudin, Annu. Rev. Fluid Mech. 45, 469 (2013).

[5] B. Andreotti, Y. Forterre, and O. Pouliquen, Cambridge University Press (2013).

[6] M. Day and G. Kocurek, Geology 46 (2018).

[7] D. E. Wilkins and R. L. Ford, Geomorphology 83, 48 (2007).

[8] N. Zgheib, J. J. Fedeld, D. C. J. D. Hoyal, M. M. Perillo, and S. Balachandar, J. Geophys. Res. 123, 478 (2018).

[9] R. D. Lorenz and J. R. Zimbelman, Springer Praxis Books (2014).

[10] P. L. Barnard, D. Hanes, D. M. Rubin, and R. Kvitek, Eos, Transactions American Geophysical Union 87, 285 (2006).

[11] S. Diniega, M. Kreslavsky, J. Radebaugh, S. Silvestro, M. Telfer, and D. Tirsch, Aeolian Research 26, 5 (2017), special Issue for the Fourth International Planetary Dunes Workshop.

[12] P. Jia, B. Andreotti, and P. Claudin, Proceedings of the National Academy of Sciences 114, 2509 (2017), https://www.pnas.org/content/114/10/2509.full.pdf.

[13] A. R. Lima, G. Sauermann, H. J. Herrmann, and K. Kroy, Physica A: Statistical Mechanics and its Applications 310, 487 (2002).

[14] E. J. R. Parteli and H. J. Herrmann, Physica A: Statistical Mechanics and its Applications 327, 554 (2003).

[15] S. Diniega, K. Glasner, and S. Byrne, Geomorphology
121, 55 (2009).

[16] E. Eastwood, J. Nield, A. Baas, and G. Kocurek, Sedimentology 58, 1391 (2011).

[17] O. Durán, P. Claudin, and B. Andreotti, Aeolian Research 3, 243270 (2011).

[18] S. Worman, A. B. Murray, R. Littlewood, B. Andreotti, and P. Claudin, Geology 41, 1059 (2013).

[19] J. H. Lee, A. O. Sousa, E. J. R. Parteli, and H. J. Herrmann, International Journal of Modern Physics C 16, 1879 (2005).

[20] M. Génois, P. Hersen, E. Bertin, S. C. du Pond, and G. Grégoire, Phys. Rev. E 94 (2016).

[21] N. Endo, K. Taniguchi, and A. Katsuki, Geophys. Res. Lett. 31 (2004).

[22] P. Vermeesch and N. Drake, Geophys. Res. Lett. 35 (2008).

[23] C. H. Hugenholtz and T. E. Barchyn, Geophys. Res. Lett. 39 (2012).

[24] K. A. Bacik, S. Lovett, C. P. Caulfield, and N. M. Vriend, Phys. Rev. Lett. 124 (2020).

[25] W. R. Assis and E. M. Franklin, Geophys. Res. Lett. 47, GL089464 (2020).

[26] A. Betat, V. Frette, and I. Rehberg, Phys. Rev. Lett. 83, 88 (1999).

[27] C. Groh, A. Wierschem, N. Aksel, I. Rehberg, and C. Kruelle, Phys. Rev. E 78, 021304 (2008).

[28] H. Mouilleron, F. Charru, and O. Eiff, J. Fluid Mech. 628, 229 (2009).

[29] J. Best, G. Blois, J. Barros, and K. Christensen, in $\mathrm{Ma}$ rine and River Dune Dynamics MARID IV (Bruges, Belgium, 2013).

[30] S. B. Dalziel, Applied Scientific Research 49, 217 (1992).

[31] S. B. Dalziel, Digiflow User Guide, Dalziel Research Partners, 4th ed. (2017). 
[32] K. Kroy, G. Sauermann, and H. J. Herrmann, Phys. Rev. Lett. 88, 054301 (2002).

[33] B. Andreotti, P. Claudin, and S. Douady, The European Physical Journal B 28, 321 (2002).

[34] H. Niiya, A. Awazu, and H. Nishimori, Phys. Rev. Lett. 108 (2012).

[35] L. Guignier, H. Niiya, H. Nishimori, D. Lague, and A. Valance, Phys. Rev. E 87 (2013).

[36] A. J. Paarlberg, C. M. Dohmen-Janssen, S. J. M. H. Hulscher, and P. Termes, Water Resour. Res. 43 (2007).

[37] I. Walker and D. Sugar, Earth Surf. Process. Landforms 38, 1642 (2013).

[38] A. D. Araújo, E. J. R. Parteli, T. Pöschel, J. S. Andrade, and H. J. Herrmann, Sci. Rep. 3 (2013).
[39] W. Anderson and M. Chamecki, Phys. Rev. E 89, 013005 (2014).

[40] M. Baddock, I. Livingstone, and G. Wiggs, Geomorphology 87, 322336 (2007).

[41] N. R. Bristow, G. Blois, J. L. Best, and K. T. Christensen, Journal of Geophysical Research: Earth Surface 124, 1175 (2019).

[42] A. W. Baar, J. de Smit, W. Uijttewaal, and M. Kleinhans, Water Resour. Res. 54 (2018).

[43] I. Bendixson, Acta Math. 24, 1 (1901).

[44] P. Hersen and S. Douady, Geophys. Res. Lett. 32 (2005).

[45] O. Durán, V. Schwämmle, and H. Herrmann, Phys. Rev. E 72, 021308 (2005). 